\title{
Accurate protein structure prediction: what comes next?
}

\author{
Chaok Seok ${ }^{1,2, \star}$, Minkyung Baek ${ }^{3}$, Martin Steinegger ${ }^{4}$, Hahnbeom Park $^{3}$, Gyu Rie Lee ${ }^{3}$ and Jonghun Won ${ }^{2}$ \\ ${ }^{1}$ Department of Chemistry, Seoul National University, Seoul 08826, Republic of Korea \\ ${ }^{2}$ Galux Inc., Seoul 08738, Republic of Korea \\ ${ }^{3}$ Department of Biochemistry and Institute for Protein Design, University of Washington, Seattle, WA 98195, USA \\ ${ }^{4}$ School of Biological Sciences, Seoul National University, Seoul 08826, Republic of Korea \\ ${ }^{*}$ Correspondence: chaok@snu.ac.kr
}

Protein structure prediction has become extremely accurate, and its results are now comparable with those of experimental methods for a large number of proteins. However, there remain some technical hurdles to clear before the current structure prediction tools can be directly applied to a wide range of biomedical problems. New perspectives on future developments in the area of structure prediction and its biomedical applications are presented.

\section{INTRODUCTION: A NEW ERA OF ACCURATE STRUCTURE PREDICTION BY ARTIFICIAL INTELLIGENCE}

Since the three-dimensional structure of myoglobin was released (Dickerson et al., 1961), protein structure determination has been an area of experimental research. Attempts to predict the threedimensional structure of proteins from their amino acid sequence began shortly thereafter, with two major academic approaches. One approach involved attempts to predict the structure based on physical laws (Rohl et al., 2004), while the other exploited experimentally determined sequence-structure relationships using informatics (Eswar et al., 2006).

While predictive methods based on physical principles are still hampered by limitations in computational complexity and lack of accuracy, a breakthrough was made using the informatics approach in 2020. In December 2020, the Critical Assessment of techniques for protein Structure Prediction (CASP) organizing committee announced that "an artificial intelligence solution to the (protein structure prediction) challenge has been found" by DeepMind's AlphaFold [https://predictioncenter.org/casp14/ doc/CASP14_press_release.html] (Kinch et al., 2021). In the wake of this exciting development, it is important to consider the following questions. Will the focus of research in structural biology pivot away from the experimental domain toward the computational domain? How will AlphaFold affect the research on structure-function relationships of bio-systems and biomedical applications in the future?

AlphaFold AND RoseTTAFold: LINKING COEVOLUTIONARY INFORMATION IN PROTEIN SEQUENCES TO PROTEIN STRUCTURE USING DEEP NEURAL NETWORKS

On July 15, 2021, two important methods were published simultaneously: the AlphaFold method (Jumper et al., 2021) and a method by Baker et al. called RoseTTAFold (Baek et al., 2021), which was inspired by AlphaFold presented at the 2020 CASP meeting (CASP14) by DeepMind. AlphaFold relies on the availability of enough sequences that are evolutionarily related to the target protein to enable accurate structure prediction. Conventional homology modeling, also called template-based modeling, requires an experimentally determined template "structure" of a related protein because the structure of the target protein is built based on the template structure using a target-template sequence alignment. Access to an available template structure is no longer a requirement with the new class of methods.

The source codes and web servers were made available along with the AlphaFold [https://github.com/deepmind/alphafold] and RoseTTAFold [https://github.com/RosettaCommons/ RoseTTAFold or https://robetta.bakerlab.org/] methods. Currently, there are two simplified versions of the AlphaFold web server available. These differ from the full AlphaFold system mostly with respect to homology detection: (1) the DeepMindbased system [https://colab.research.google.com/github/ deepmind/alphafold/blob/main/notebooks/AlphaFold.ipynb] uses HMMer to search a reduced sequence set, and (2) a version developed by researchers from Harvard University, Max Planck Institute, and Seoul National University [https://colab.research. google.com/github/sokrypton/ColabFold/blob/main/AlphaFold2. ipynb] speeds up the alignment generation by replacing HMMer with MMseqs2 and other additional features (Mirdita et al., 2021).

Evolutionarily related protein sequences contain information about the coevolution process between amino acids that have been forced to maintain physical interactions to form a specific structure for their function. Attempts to extract coevolutionary information for predicting which amino acid pairs make physical contact in the 3D structure began as early as the late 1990s (Lesk, 1997). This type of coevolution-based contact prediction resulted in a dramatic improvement in protein structure prediction accuracy in CASP12 (Abriata et al., 2018). The nextstage of development exploiting coevolutionary information was to use deep learning, as introduced by several groups in CASP13 
(Abriata et al., 2019). The deep-neural-network architectures of both AlphaFold and RoseTTAFold have effectively linked this coevolution information to the protein structure to produce highly accurate protein models, as illustrated in CASP14 and related benchmark tests.

\section{ACCURATE PROTEIN STRUCTURE PREDICTION: LIMITA- TIONS AND OPPORTUNITIES}

It is very impressive that a large amount of biological data allowed an informatics-based method to succeed while a physics-based method could not. This success was mostly due to the rapid advances in next-generation sequencing technology that have increased the available protein "sequence" data. The remarkable progress in protein structure determination methods also provided sufficient protein "structure" data to train deeplearning-based structure prediction methods.

However, the AlphaFold and RoseTTAFold methods are less effective when insufficient information is available. In other words, accurate structure prediction cannot be realized when no or few evolutionarily related sequences for the target protein are available, at least for now. For example, if a variety of evolved protein sequences, such as those of bacterial proteins, are known, the predicted structures will be accurate. However, for some proteins, the structure prediction results may not be as reliable because of the lack of diversity in known sequences. The normalized number of effective sequences, called Neff, is an important variable that affects the structural prediction accuracy of both AlphaFold and RoseTTAFold. For example, at present, $N_{\text {eff }}>30$ may be considered a rough criterion for accurate prediction (Baek et al., 2021; Jumper et al., 2021). Both AlphaFold and RoseTTAFold provide the estimated structure accuracy for each amino acid, which can be utilized to interpret experimental results or to propose or validate hypotheses.
In the RoseTTAFold paper, several application examples of accurately predicted structures were demonstrated such as crystal structure determination by molecular replacement, analysis of cryo-EM structure, and explanation of structurebased protein functions. Further applications, such as structurebased drug discovery, could be possible if molecular interactions between proteins and drug molecules can be accurately predicted. Protein sequences have evolved conserving interactions with other biomolecules as well as intra-protein structures to maintain proper functions. Therefore, the AlphaFold and RoseTTAFold methods or the fundamental ideas behind them may be utilized to predict complex structures of proteins and other molecules in cases where sufficient data exist. In the future, we hope that more accurate structure predictions of complexes involving proteins such as protein-protein, proteinnucleic acid, and protein-compound complexes will be realized.

Notably, Baek et al. (2021) demonstrated that RoseTTAFold could be used to predict protein-complex structures from evolutionarily related sequence pairs of two different proteins that interact in the same species. AlphaFold has also been observed to accurately predict complex structures in some cases when sequence pairs of two interacting proteins are provided with proper relative positional information. The prediction of proteinpeptide complex structures was less successful. However, the full scope of this new technique and the speed with which accurate protein complex structure predictions are possible are yet to be revealed.

In the CASP competition held in 2020, AlphaFold was able to generate high-accuracy subunit structures for homooligomer targets that could be used to build the full oligomer structure through simple geometric combinations (Kinch et al., 2021). This implies that AlphaFold accounts for oligomer contexts when predicting monomer structures. In one instance,

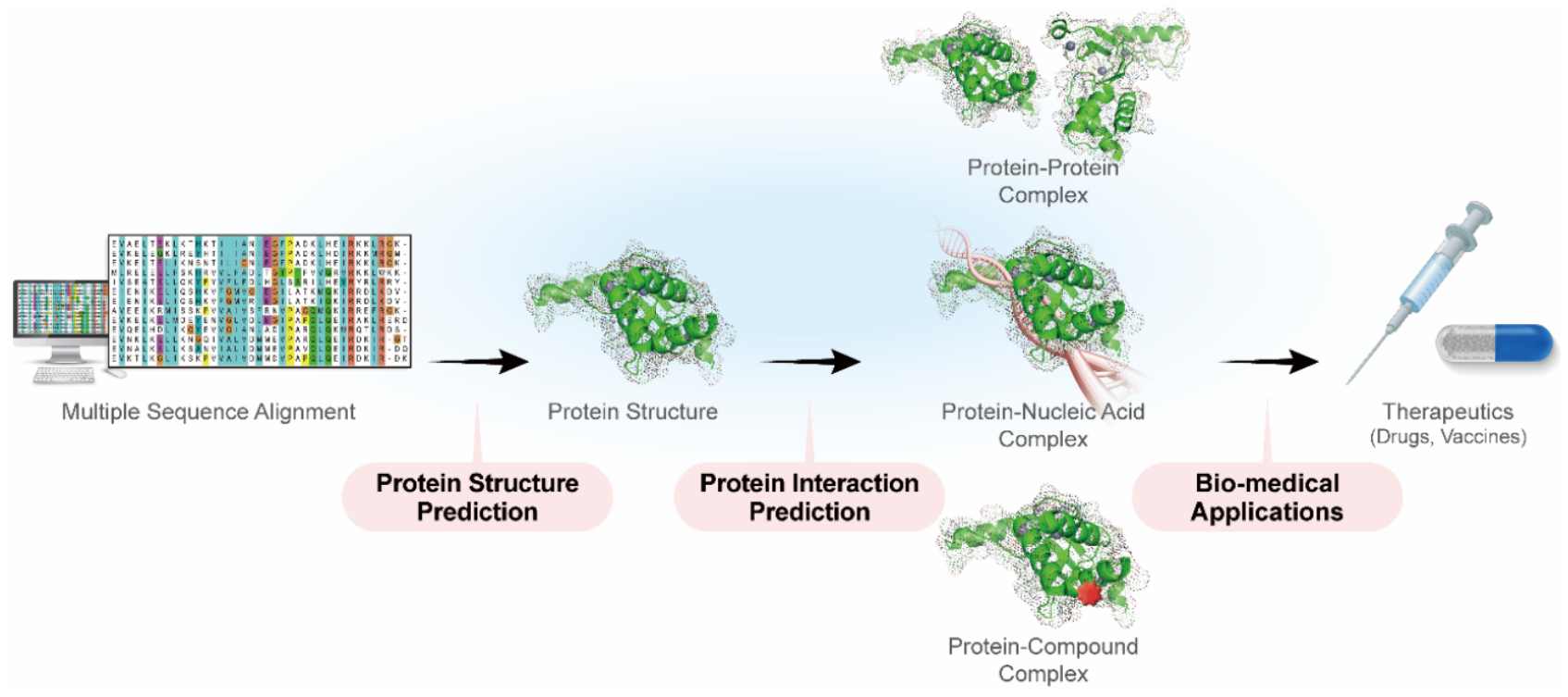


AlphaFold mistakenly incorporated a local feature of the intersubunit structure into the intra-subunit structure in CASP14. This interesting example implies that inter-subunit structural information in the sequences of related proteins can be extracted by the current network model, which means that accurate oligomer structure prediction incorporating such information may soon be possible.

Methods for both hetero- and homo-oligomer structure prediction have been tested in the assembly category of the CASP and CAPRI (Critical Assessment of PRotein Interactions) competitions. Oligomer versions of AlphaFold and RoseTTAFold are expected to be entered in the upcoming 2022 CASP and CAPRI competitions. Depending on the types and the number of subunits required to form an oligomer, a variety of symmetrical structures are theoretically possible. How well such complex structures can be predicted would be an interesting point to examine in the next competition.

\section{BARRIERS TO ACCURATE PREDICTION FOR FUTURE BIOMEDICAL APPLICATIONS}

Even with the remarkable breakthrough for structure prediction made in CASP14, in which experimentally well-resolved protein structures are compared with the predicted structures, it is likely that accurate prediction will be more difficult for proteins whose structural changes are wholly or partially dependent on their influences from their surroundings, including interacting partner molecules, chemical modifications of some amino acids and acidity. If the information on structural changes is incorporated in the evolutionarily related protein sequences, a neural network architecture could be designed to extract such information, and prediction of some structural changes could become possible in the near future. It remains unclear how accurately the structure of an intrinsically disordered protein or a dynamically changing protein can be predicted based on an information-based deeplearning method.

Another point to consider is the accuracy of the technique for predicting the structure of human-designed proteins that are not generated by natural evolution. The Rosetta community has already begun to use structural prediction methods based on coevolution in protein design (Norn et al., 2021). However, more research is required to determine its usefulness and limitations.

Interactions of proteins with DNA or RNA are represented to some extent in the structure database; thus, predicting the structure of such complexes could be attempted. It is expected that such a method would be less successful than protein structure prediction because of the limited amount of proteinnucleic acid complex sequence and structure information available. Predictions of the interactions between proteins and small molecules are of particular use for the field of drug development, so many new attempts are likely to be made. However, research in these areas will require more imagination and creativity due to the difficulty of transforming various kinds of existing information into structural and interaction properties.
For example, representing chemicals with diverse building blocks on a computer is more complicated than representing proteins that consist of only 20 types of amino acids.

To understand interactions of proteins and other molecules, researchers need to know not only the complex structures but also how the structural interactions are linked to the activity of proteins. However, currently available data related to activity is much smaller than that related to structure. Moreover, a data related to activity, for example, the binding strength of a single protein-protein complex comprises a very small amount of information compared to a complex structure itself, owing to the large number of interactions between different amino acids in complex structures. A much larger collection of binding affinity or activity data for numerous amino acid variations would be necessary to provide useful information for the accurate prediction of binding strength and activity.

\section{SPECULATIONS FOR FUTURE DEVELOPMENT IN PROTEIN MODELING BY ARTIFICIAL INTELLIGENCE}

Due to the nature of artificial intelligence, it is difficult to interpret what exactly has been learned. What have the neural network models for protein structure prediction learned from the large numbers of protein structures and evolution patterns in sequences? Something that corresponds to physical principles, perhaps? Could they then provide something useful from the physical principle they learned even when there is not enough direct information for a particular bio-system? If not, stronger prediction power could be gained by learning from more experimental data and accurate prediction data, and protein structure research could shift from being experimental to being mostly computational and predictive.

Protein structure prediction has become extremely accurate, and its results are now comparable with those of experimental methods for a large number of proteins. However, there remain some technical hurdles to clear before the current structure prediction tools can be directly applied to a wide range of biomedical problems. In particular, it remains unclear how accurately molecular interactions, in terms of both complex structures and interaction strengths, will be predicted. For the time being, experimental research is likely to remain key in many cases, including bio-systems involving proteins that have insufficient evolutionarily related sequences, proteins that vary in structure depending on binding partners or environment, and dynamic proteins.

\section{ACKNOWLEDGEMENTS}

The work was supported by the National Research Foundation of Korea (NRF) grant funded by the Korea government (No. 2020M3A9G7103933).

\section{CONFLICT OF INTEREST}

The authors declare no conflicts of interest. 
Original Submission: Aug 4, 2021

Revised Version Received: Aug 20, 2021

Accepted: Aug 20, 2021

\section{REFERENCES}

Abriata, L.A., Tamò, G.E., and Dal Peraro, M. (2019). A further leap of improvement in tertiary structure prediction in CASP13 prompts new routes for future assessments. Proteins 87, 1100-1112.

Abriata, L.A., Tamò, G.E., Monastyrskyy, B., Kryshtafovych, A., and Dal Peraro, M. (2018). Assessment of hard target modeling in CASP12 reveals an emerging role of alignment-based contact prediction methods. Proteins 86 Suppl 1, 97-112.

Baek, M., DiMaio, F., Anishchenko, I., Dauparas, J., Ovchinnikov, S., Lee, G.R., Wang, J., Cong, Q., Kinch, L.N., Schaeffer, R.D., Millán, C., Park, H., Adams, C., Glassman, C.R., DeGiovanni, A., et al. (2021). Accurate prediction of protein structures and interactions using a three-track neural network. Science 373, 871-876.

Dickerson, R.E., Kendrew, J.C., and Strandberg, B.E. (1961). The crystal structure of myoglobin: phase determination to a resolution of $2 \AA$ by the method of isomorphous replacement. Acta Cryst 14, 1188-1195.

Eswar, N., Webb, B., Marti-Renom, M.A., Madhusudhan, M.S., Eramian,
D., Shen, M.Y., Pieper, U., and Sali, A. (2006). Comparative protein structure modeling using Modeller. Curr Protoc Bioinformatics Chapter $\mathbf{5}$, Unit-5.6.

Jumper, J., Evans, R., Pritzel, A., Green, T., Figurnov, M., Ronneberger, O., Tunyasuvunakool, K., Bates, R., Žídek, A., Potapenko, A., Bridgland, A., Meyer, C., Kohl, S.A.A., Ballard, A.J., Cowie, A., et al. (2021). Highly accurate protein structure prediction with AlphaFold. Nature 596, 583589.

Kinch, L.N., Pei, J., Kryshtafovych, A., Schaeffer, R.D., and Grishin, N.V. (2021). Topology evaluation of models for difficult targets in the 14th round of the critical assessment of protein structure prediction. Proteins doi: 10.1002/prot.26172. [Epub ahead of print].

Lesk, A.M. (1997). CASP2: report on ab initio predictions. Proteins Suppl $1,151-166$.

Mirdita, M., Ovchinnikov, S., and Steinegger, M. (2021). ColabFoldMaking protein folding accessible to all. bioRxiv https://doi. org/10.1101/2021.08.15.456425.

Norn, C., Wicky, B.I.M., Juergens, D., Liu, S., Kim, D., Tischer, D., Koepnick, B., Anishchenko, I., Baker, D., and Ovchinnikov, S. (2021). Protein sequence design by conformational landscape optimization. Proc Natl Acad Sci U S A 118, e2017228118.

Rohl, C.A., Strauss, C.E., Misura, K.M., and Baker, D. (2004). Protein structure prediction using Rosetta. Methods Enzymol 383, 66-93. 